ASSOCIATION BETWEEN METABOLIC SYNDROME AND NUTRITION, SLEEP AND PHYSICAL ACTIVITY IN CHILDREN

doi:10.1136/archdischild-2012-302724.0366

'S Jalilolghadr, ${ }^{2 \mathrm{M}}$ Javadi, ${ }^{2} \mathrm{M}$ Farshidgohar, ${ }^{2} \mathrm{~A}$ Javadi. ${ }^{1}$ Pediatrics, Qazvin University of Medical Sciences; ${ }^{2}$ Qazvin University of Medical Sciences, Oazvin, Iran

Background and Aims Metabolic syndrome includes a collection of risk factors that increase the risk of cardiovascular diseases and diabetes type 2. The prevalence of both conditions has been increased due to urbanization and changes in life style. The purpose of this study was to determine the prevalence of metabolic syndrome in children and its relationship with nutritional habits sleep duration and physical activity.

Methods A cross-sectional study was conducted on 338 (166 male, 172 female) children aged 10-18 years old. Waist circumference, blood pressure, blood sugar and lipid profile were measured. Metabolic syndrome defined according to IDF criteria. The pattern of nutrition, physical activity and sleep evaluated with Iranian version of international valid questionnaires.

Results Prevalence of metabolic syndrome was $11.8 \%$. There was no significant association between sleep duration and metabolic syndrome except association between short sleep duration and high level of LDL $(\mathrm{P}<0.03)$. The average daily salt consumption of in metabolic syndrome patients was more than healthy children. Subjects with daily active activity more than 2 hours had lower level of LDL and metabolic syndrome prevalence $(p=0.04)$. Metabolic syndrome was significantly lower in subjects with organized activity.

Conclusion Metabolic syndrome is highly prevalent in Iranian children. These findings suggest importance of healthy nutritional habits and organized physical activity to prevent metabolic syndrome.

\section{POST RESUSCITATIVE FACTORS INFLUENCING SURVIVAL AFTER IN-HOSPITAL PEDIATRIC CARDIAC ARREST}

doi:10.1136/archdischild-2012-302724.0367

1.2 J del Castillo, 1,2 J López-Herce, European-Latinoamerican Study Group of Cardiac Arrest in Children. ${ }^{1}$ Hospital General Universitario Gregorio Marañón; ${ }^{2}$ Red Iberoaemericana de Estudio de la Parada Cardiaca en la Infancia, Madrid, Spain

Background and Aims In-hospital pediatric cardiac arrest (CA) achieves high return of spontaneous circulation (ROSC) rates but survival to hospital discharge is low. The aim of this study was to determine post-resuscitative features that may influence survival.

Methods Prospective, international, observational, multicentric study. CA in children between 1 month and 18 years were analyzed using the Utstein template. Post-resuscitative clinical features after achieving sustained ROSC that correlated with survival to discharge were analyzed.

Results 563 episodes of in-hospital CA in 502 patients were evaluated. $346(68.9 \%)$ achieved ROSC for at least 20 minutes. 180 of them $(52 \%)$ survived to hospital discharge. Cardiac rhythm at the time of ROSC was sinus rhythm in $56.5 \%$ of the survivors and $43.5 \%$ of non survivors ( $p=0.03$ ). $74 \%$ of the patients were mechanically ventilated and $68.7 \%$ received inotropic drugs with no statistically significant differences observed between survivors and non survivors. Survivors received significantly lower mean dopamine (10.2 vs $13.4 \mathrm{mcg} / \mathrm{kg} / \mathrm{min} ; \mathrm{p}=0.002)$ and dobutamine (10.1vs 12.3 $\mathrm{mcg} / \mathrm{kg} / \mathrm{min} ; \mathrm{p}=0.03$ ) doses. Patients who survived had significantly higher mean $\mathrm{pH}$ (7.26 vs 7.17; $\mathrm{p}=0.02)$, and $\mathrm{HCO} 3$ (20.2 vs 18.4; $\mathrm{p}=0.04)$, and lower base excess ( $-5.6 \mathrm{vs}-8.2 \mathrm{p}=0.02)$ and lactic acid (5.3 vs $7.7 ; p=0.006$ ) values one hour after ROSC. Patients who survived had higher mean $\mathrm{PO} 2$ values and lower mean $\mathrm{PCO} 2$ values, but these differences did not achieve statistical signification.
Conclusion In-hospital pediatric CA, the post ROSC factors associated with mortality were cardiac rhythm, metabolic acidosis and doses of inotropic drugs.

\section{MARKERS FOR INVASIVE BACTERIAL INFECTION IN WELL- APPEARING YOUNG FEBRILE INFANTS. THE VALUE OF PROCALCITONIN}

doi:10.1136/archdischild-2012-302724.0368

1.2S Mintegi, ${ }^{3} \mathrm{~S}$ Bressan, ${ }^{1} \mathrm{~B}$ Gomez, ${ }^{4} \mathrm{~L}$ Da Dalt, ${ }^{5} \mathrm{D}$ Blázquez, ${ }^{6} \mathrm{O}$ Olaciregui, ${ }^{7} \mathrm{M}$ De La Torre, ${ }^{8} \mathrm{M}$ Palacios, ${ }^{3 P}$ Berlese, ${ }^{9} \mathrm{~A}$ Ruano. 'Pediatric Emergency, Cruces University Hospital; ${ }^{2}$ Department of Pediatrics, University of the Basque Country, Bilbao, Spain; ${ }^{3}$ Pediatrics, University of Padova, Padova; ${ }^{4}$ Ospedale Ca'Foncello, Department of Pediatrics, Treviso, Italy; ${ }^{5}$ Department of Pediatrics, 12 de Octubre University Hospital, Madrid; ${ }^{6}$ Pediatric Emergency, Donostia University Hospital, Donostia; ${ }^{7}$ Pediatric Emergency, Niño Jesús University Hospital, Madrid; ' ${ }^{8}$ ediatric Emergency, Navarra University Complex, Pamplona: ${ }^{9}$ Pediatric Emergency, Basurto University Hospital, Bilbao, Spain

Background In the last decade, the procalcitonin (PCT) has been introduced in many protocols for the management of the febrile child. However, its value among young well-appearing infants is not completely defined.

Objective To assess the value of PCT in diagnosing serious bacterial infections and specifically invasive bacterial infections (IBIs) in well-appearing infants under 3 months of age with fever without source (FWS).

Design and Methods Retrospective study including well-appearing infants under 3 months of age with FWS attended in seven European Paediatric Emergency Departments. An IBI was defined when a bacterial pathogen was isolated in blood or cerebrospinal fluid culture.

Results A total of 1,531 infants under 3 months of age with FWS were attended. There were 1,112 well-appearing infants in whom PCT and a blood culture were performed. Among them, 23 (2.1\%) were diagnosed with an IBI. A multivariate analysis showed that, among different epidemicological data and blood tests, PCT was the only independent risk factor for having an IBI (OR 21.69 if PCT 0.5 $\mathrm{ng} / \mathrm{mL}$ ). Comparing with C-Reactive Protein, PCT showed a better performance to rule-in an IBI. Among patients with normal urine dipstick and short-evolution fever (less than 6 hours), areas under the ROC curve were 0.819 and 0.563 , respectively for detecting IBIs. Conclusions Among young infants with FWS, PCT showed a better performance than C-Reactive Protein in identifying patients with IBIs and, mainly in those patients with normal urine dipstick and short-evolution fever, PCT seems to be also the best marker to rule out an IBI.

\section{EVALUATION OF CARBON MONOXIDE POISONING IN CHILDREN}

doi:10.1136/archdischild-2012-302724.0369

H Boztepe, Z Yalaki, Y Dallar Bilge. Ankara Research and Training Hospital, Ankara, Turkey

Carbon monoxide poisoning is an important health problem that may lead to deaths during winters. The purpose of this study was to patients who were diagnosed with carbon monoxide poisoning.

Methods 196 patients between 0-17 years of age, who were treated at the Ankara Education and Research Hospital Child Emergency Service for the carbon monoxide poisoning diagnosis between November 2011 and April 2012 were evaluated. Patients' complaints, socio-demographic data, biochemical parameters were recorded.

Findings The age median of the patients was 10.0 while $51.5 \%$ were female. The most frequent complaint was: nausea, vomiting (27.6\%). Carboxyhemoglobin (COHb) level average was $25.5 \pm 10.3$ 